\title{
Noninvasive Measure of Microvascular Nitric Oxide Function in Humans Using Very Low-Frequency Cutaneous Laser Doppler Flow Spectra
}

JULIAN M. STEWART, MD, PHD, INDU TANEJA, MICHAEL S. GOLIGORSKY, MD, PHD, and MARVIN S. MEDOW, PHD New York Medical College. Valhalla, New York, USA

\begin{abstract}
Objective-While higher frequency oscillations $(0.021-0.6 \mathrm{~Hz})$ in cutaneous blood flow measured by laser Doppler flowmetry (LDF) relate to oscillations in blood pressure and sympathetic nerve activity, very low-frequency oscillations (VLF, 0.0095-0.021 Hz) do not. The authors investigated whether VLF LDF power is nitric oxide (NO) specific.

Methods-LDF combined with intradermal microdialysis was used in the calves of 22 healthy volunteers aged 19-27 years. LDF power spectral analysis was performed by windowed fast Fourier transform. The authors tested whether the NO synthesis inhibitor nitro-l-arginine (NLA) produced selective decreases in VLF power before and after stimulation with acetylcholine.
\end{abstract}

Results-NLA alone did not alter total power but selectively reduced VLF power by approximately 50\%. LDF and spectral power increased markedly across all spectra with acetylcholine. This increase was blunted by NLA, which selectively reduced VLF power by approximately $50 \%$.

Conclusions-The data suggest that VLF oscillations in the laser Doppler signal are NO dependent, increase with cholinergic stimulation, and have potential as a noninvasive marker for NO-dependent microvascular reactivity.

\section{Keywords}

acetylcholine; lasers; nitric oxide; skin

\begin{abstract}
The rhythmic contraction of vascular smooth muscle, also known as vasomotion, is characteristic of all vascular beds [1]. In larger arterial vessels this manifests as HeringTraube-Mayer [2] waves in which arterial pressure fluctuates with a central frequency of approximately $0.1 \mathrm{~Hz}$. Similar $0.1-\mathrm{Hz}$ oscillations are noted in the heart rate, which are believed to be coherently transduced from pressure oscillations through the agency of the vagal arm of the baroreflex [3]. Fluctuations in pressure and heart rate are also affected by respiration and therefore a second "high"-frequency peak in the variability of these signals is
\end{abstract}

Address correspondence to Julian M. Stewart, MD, PhD, Professor of Pediatrics and Physiology, The Center for Pediatric Hypotension, Suite 3050, 19 Bradhurst Avenue, New York Medical College, Hawthorne, NY 10532, USA. stewart@nymc.edu. 
observed at approximately $0.25-0.30 \mathrm{~Hz}$, corresponding to the respiratory sinus arrhythmia [4].

Oscillations in cutaneous blood flow are also apparent during laser Doppler flowmetry (LDF) [5], and coherence analysis has attributed this LDF "flowmotion" to microvascular vasomotion [6,7]. Detailed analyses by Bernardi and colleagues [8] have demonstrated that "low"-frequency LDF fluctuations are coherent with systolic arterial blood pressure, muscle sympathetic nerve activity (MSNA), and R-R interval, while "high"-frequency LDF fluctuations are coherent with systolic arterial blood pressure, MSNA, R-R interval, and respirations, and strongly relate to skin sympathetic nerve activity [9]. Indeed, similar peaks are found in sympathetic nerve activity in both muscle [10] and skin [11], and correspond to the low-frequency Mayer waves and higher-frequency respiratory sinus arrhythmia.

It is therefore interesting that spectral analysis of cutaneous blood flow oscillations often demonstrates a distinct third peak at very low frequency $(<0.21 \mathrm{~Hz})[12-15]$. This peak has no counterpart in MSNA, R-R interval, respiratory, or blood pressure power spectra and is incoherent with systolic blood pressure and other signals; thus, it is unrelated to central or peripheral neurological activity. One might infer that it represents contributions to LDF oscillations arising from local mechanisms that may be endothelial mediated. Support for endothelial dependence of the very low-frequency LDF oscillations originates with the work of Kvernmo using acetylcholine iontophoresis [14, 15], although data were not definitive in untrained control subjects and were potentially confounded by the vasodilator properties of anodal current [16], which evokes a C-fiber-dependent axon reflex [17]. Also, acetylcholinedependent microvascular vasodilation in diverse tissues is axon reflex dependent [18] and may relate to other signaling molecules (e.g., prostaglandins [19] and endothelial-dependent hyperpolarizing factor, EDHF, [20]). Thus, microvascular simulation of cholinergic receptors does not clearly separate nitric oxide (NO) from other molecular contributions.

We hypothesize, therefore, that very low-frequency power of LDF is highly NO specific. To test this hypothesis we examined LDF flowmotion using the technique of intradermal microdialysis to deliver a nitric oxide synthase (NOS) inhibitor, nitro-L-arginine (NLA) before and after receptor mediated dilation with acetylcholine.

\section{MATERIALS AND METHODS}

Experiments were performed on two separate days in each subject. All drugs were administered through microdialysis catheters. On one day we measured baseline LDF and LDF power spectra over a given site before and after insertion of a microdialysis catheter to determine whether catheter insertion changes the flow and spectra. This comprised protocol 1. Then we determined the effects of the non-isoform-specific NOS inhibitor nitro-L-arginine on LDF and laser Doppler spectral power. This comprised protocol 2.

On another day we measured baseline LDF and spectral power in the skin over the microdialysis catheter, then repeated these measurements after acetylcholine (AcCh) administration. This comprised protocol 3. Finally, we determined the effects of NLA on 
LDF and laser Doppler power spectra during steady acetylcholine administration. This comprised protocol 4.

Experimental days were presented in random order.

\section{Subjects}

We studied 22 healthy volunteers aged $19-27$ years (mean $=23.9 \pm 0.6$ years). There were 8 men and 14 women. Only subjects found to be free from systemic diseases and cardiovascular disease were eligible. No subjects were taking neurally active or vasoactive medications. Subjects refrained from alcohol or caffeinated beverages for $24 \mathrm{~h}$ prior to study. There were no smokers or trained competitive athletes. Informed consent was obtained and the Committee for the Protection of Human Subjects (IRB) of New York Medical College approved all protocols.

\section{Instrumentation}

All testing was conducted in a temperature-controlled room (approximately $25^{\circ} \mathrm{C}$ ) at least 2 $\mathrm{h}$ after a light breakfast. Experiments began after a 30-min acclimatization period. All experiments were performed while the subject remained supine and breathing spontaneously. Measurements were made in the left calf. Skin temperature was measured with a thermal probe to ensure neutral thermal conditions. An integrating laser-Doppler flow-probe (Probe 413, Perimed, Stockholm) containing 7 individual elements was placed over a prospective microdialysis site on the lateral aspect of the left calf. We measured LDF for approximately $15 \mathrm{~min}$ using the last $10 \mathrm{~min}$ for analysis. The positions of the lasers were marked, lasers were removed, and microdialysis probes were inserted in the dermal space of the insertion site. Each probe comprised a 10-mm microdialysis membrane window placed in the intradermal space using a 25-gauge needle as an introducer and with the tip of the needle exiting the skin approximately $2 \mathrm{~cm}$ from the point of insertion. We threaded the microdialysis probe (LM-10 Linear Microdialysis Probes, Bioanalytical Systems, West Lafayette, IN) through the inserter needle. The probe has a nominal molecular weight cutoff of $10,000 \mathrm{Da}$. We used markers to facilitate central positioning of the dialysis membrane. The needle was removed, with the centrally located $10-\mathrm{mm}$ window left in place. Catheters were initially perfused with Ringer's solution at $2 \mu \mathrm{L} / \mathrm{min}$. The leg was maintained at the level of the heart throughout all procedures. The integrating laser-Doppler flow-probe was replaced directly over each dialysis probe to measure LDF during recovery. Recovery lasted for at least $60 \mathrm{~min}$ and usually 90-120 min, until the LDF returned to baseline levels [21]. All laser Doppler flows were measured in arbitrary perfusion units (pfu). Drugs, when used, were delivered by microdialysis at a constant perfusion rate of $2 \mu \mathrm{L} / \mathrm{min}$ throughout experiments. Continuous LDF data were collected at a sampling rate of $200 \mathrm{~Hz}$ during experiments, multiplexed, and interfaced to a personal computer through an A/D converter (DI-720, DATAQ Industries, Milwaukee, WI) using custom data acquisition software, and generating binary files and computer displays of simultaneously collected data from all lasers (and blood pressure data) within a directory for a given patient. 


\section{Protocol 1: The Effects of Microdialysis Catheter Insertion on Baseline Flow and LDF Power Spectra}

The purpose of this experiment was to determine whether, on the average, instrumentation with microdialysis catheters affect LDF and LDF spectra. After the 30-min acclimatization period subjects had baseline LDF data collected for at least $10 \mathrm{~min}$. Laser probes were then removed and the microdialysis catheter inserted. During the ensuing recovery, LDF recovered on average to precatheterization levels. For this protocol all catheters contained Ringer's solution perfusate delivered at a rate of $2 \mu \mathrm{L} / \mathrm{min}$ while LDF monitoring continued. After recovery, we remeasured laser Doppler flow and LDF flow spectra during the last 10 min of a 30-min baseline period.

\section{Protocol 2: The Effects of NLA on LDF, and LDF Spectra}

The purpose of this experiment was to test the hypothesis that NOS inhibition reduces very low-frequency spectral power. After recovery, patients had baseline LDF data collected for at least $10 \mathrm{~min}$. Subjects then received perfusate containing $10 \mathrm{mM}$ NLA at a rate of 2 $\mu \mathrm{L} / \mathrm{min}$ while LDF monitoring continued for $30 \mathrm{~min}$. NLA was dissolved in Ringer's solution. For purposes of analysis only the last $10 \mathrm{~min}$ of data were used. Steady-state LDF flows and power spectra were achieved by this time.

\section{Protocol 3: The Effects of Acetylcholine on LDF and LDF Spectra}

The purpose of this experiment was to examine the overall effects of receptor-mediated stimulation with acetylcholine on LDF and LDF power spectra. After recovery, patients had baseline LDF data collected for at least $10 \mathrm{~min}$. Subjects then received perfusate containing $100 \mathrm{mM}$ AcCh dissolved in Ringer's solution through each of catheters 1, 2, and 3 at a rate of $2 \mu \mathrm{L} / \mathrm{min}$ while LDF monitoring continued for 30 mins. For purposes of analysis only the last $10 \mathrm{~min}$ of data were used.

\section{Protocol 4: The Effects of NLA on Acetylcholine-stimulated LDF and LDF Spectra}

Acetylcholine stimulates a variety of endothelial vasoactive substances. The purpose of this experiment was to test the hypothesis that NOS inhibition specifically reduces very lowfrequency spectral power following AcCh stimulation. Subjects of protocol 3 had LDF data collected for at least 10 min while receiving acetylcholine by microdialysis. Subjects then received perfusate containing $100 \mathrm{mM}$ AcCh $+10 \mathrm{mM}$ NLA dissolved in Ringer's solution and administered at a rate of $2 \mu \mathrm{L} / \mathrm{min}$ while LDF monitoring continued for $30 \mathrm{~min}$. For purposes of analysis only the last 10 min of data were used.

\section{Monitoring}

Heart rate was monitored by electrocardiography and right upper extremity blood pressures were measured by finger plethysmography intermittently recalibrated against oscillometry in the right arm. Mean arterial pressure (MAP) was calculated as the sum of the (systolic pressure $+2 *$ diastolic pressure) $/ 3$. 


\section{Fourier Analysis}

All processing was performed using custom software that we have used previously [22]. Ectopy was corrected by removing the ectopic beat and interpolating with a cubic spline for 5 beats in either direction. This was rarely necessary. A Butterworth digital filter was applied to sampled data to effectively remove frequencies above $0.6 \mathrm{~Hz}$. This removed aliasing from the heart rate frequencies, which was excluded from analysis. Hamming windowed fast Fourier transform (FFT) was performed, digital power spectra calculated from the squared amplitude at each frequency to generate a spectral power density function, and spectral power summed at each frequency within a given frequency band. Spectral power was partitioned into very low frequency of $0.0095-0.021 \mathrm{~Hz}$, low frequency of $0.021-0.052 \mathrm{~Hz}$, midrange $0.052-0.145 \mathrm{~Hz}$ and high frequency $0.145-0.6 \mathrm{~Hz}$ [14]. The "total power" was also calculated as the sum over all bands and used as a denominator to obtain normalized power in a given band.

\section{Data and Statistical Analysis}

We observed that LDF and LDF spectral power can vary widely from microdialysis site to microdialysis site on the calf (and forearm) despite the use of integrating laser Doppler probes. This was independent of distal-proximal location. Even marking the particular LDF site, then lifting the LDF probe off the site and then reapplying the probe to the same site can yield substantially different LDF magnitudes over the very same location. Thus, while we present absolute LDF and power spectral data, we primarily analyzed responses to drugs in terms of percent change of paired comparisons for each microdialysis catheter - LDF site whose location is unperturbed throughout the course of experiments. Thus, each catheter served as its own control. However, we now present data indicating that, on average, instrumentation per se does not affect the outcome of experiments.

Paired comparisons are made throughout. In protocol 1 the comparison is between baseline flow and spectra before insertion of the microdialysis catheter and baseline flow and spectra after insertion of the microdialysis catheter. In protocol 2 the comparison is between baseline flow and spectra after insertion of the microdialysis catheter and flow and spectra after NLA has been administered. In protocol 3 the comparison is between baseline flow and spectra after insertion of the microdialysis catheter and flow and spectra after acetylcholine has been administered. In protocol 4 the comparison is between flow and spectra after acetylcholine alone has been administered and flow and spectra after NLA + acetylcholine has been administered.

Text and graphic results are reported as means \pm standard errors of the mean. Changes in laser Doppler flow and in LDF total spectral power before and after NLA were compared by paired $t$ tests. We used two-way ANOVA with repeated measures to compare normalized (designated "normed" on graphs) and unnormalized (designated "absolute" on graphs) LDF power spectral data as a function of frequency. Results were calculated using SPSS (Statistical Package for the Social Sciences) software version 11.0. 


\section{RESULTS}

Male subjects were larger than female subjects (weight $=84.0 \pm 4.2$ vs. $63.2 \pm 2.4 \mathrm{~kg}$, height $=178 \pm 2$ vs. $166 \pm 2 \mathrm{~cm}, \mathrm{BMI}=26.6 \pm 1.4$ vs. $\left.23.2 \pm 1.0 \mathrm{~kg} / \mathrm{m}^{2}\right)$. Supine heart rate and blood pressure were not different in males compared to females (HR $=58 \pm 3$ vs. $63 \pm 2$ bpm, systolic $\mathrm{BP}=122 \pm 2$ vs. $117 \pm 3 \mathrm{mmHg}, \mathrm{MAP}=87 \pm 2$ vs. $83 \pm 2 \mathrm{mmHg}$ ).

\section{Protocol 1: The Effects of Microdialysis Catheter Insertion on Baseline Flow and LDF Power Spectra}

The effects of microdialysis catheter insertion are depicted in Figure 1. There is no significant change in laser Doppler flow and the flow spectra are similar fora representative subject. Once recovered from the trauma of instrumentation, there is on average no effect of catheter insertion on LDF power spectra.

\section{Protocol 2: The Effects of NLA on LDF, and LDF Spectra}

When we averaged baseline postcatheter recovery data over all probes before any drugs were given, we found that $23 \pm 2 \%$ of total power was contained in very low-frequency band $(0.0095-0.021 \mathrm{~Hz}), 36 \pm 3 \%$ of total power was contained in the low-frequency band $(0.021-0.052 \mathrm{~Hz}), 34 \pm 3 \%$ was contained in the mid-frequency band $(0.052-0.145 \mathrm{~Hz})$, and $8 \pm 2 \%$ was contained in the high-frequency band $(0.145-0.6 \mathrm{~Hz})$. Baseline data and the effects of NLA on absolute laser Doppler blood flows (LDF), expressed in perfusion units (pfu), and total spectral power summed over all frequency bands, expressed in units of $\mathrm{pfu}^{2}$, are shown in Figure 2. NLA had no significant effect on laser Doppler flow or total spectral power.

Figure 3 shows the effect of NLA on the frequency distribution of spectral power in a representative subject. There is a reduction in very low frequency power but little change in other spectra after NLA. Figure 4 shows the averaged effects of NLA absolute and normalized spectral power within each frequency band. NLA selectively $(p<.001)$ reduced normalized and absolute power by approximately $50 \%$ for very low frequencies only.

\section{Protocol 3: The Effects of Acetylcholine on LDF and LDF Spectra}

Laser Doppler flow and total spectral power increased significantly (from $18 \pm 3$ to $126 \pm 11$ pfu, and from $3.7 \pm 0.8$ to $20.9 \pm 3.0 \mathrm{pfu}^{2}, p<.0001$ ) with the perfusion of acetylcholine. As shown in Figure 5, there was no change in normalized power at any frequency band. Thus, the percent increase in spectral power was evenly distributed over all frequencies.

\section{Protocol 4: The Effects of NLA on Acetylcholine-stimulated LDF and LDF Spectra}

The effects of NLA on AcCh-stimulated laser Doppler flow and on total spectral power are shown in Figure 6. LDF and overall spectral power were significantly reduced by NLA ( $p$ $<.05)$. The reduction in LDF and in total spectral power did not reduce flow or spectral power to baseline, pre-acetylcholine values. 
Figure 7 shows the effects of acetylcholine combined with NLA on absolute and normalized frequency distribution of spectral power. NLA selectively $(p<.001)$ reduced normalized power by approximately $50 \%$ for very low frequencies only.

\section{DISCUSSION}

The major finding of our study is that NLA, a nitric oxide synthase inhibitor, reduces very low-frequency oscillations in the laser Doppler power spectrum both before and after cholinergic receptor-mediated stimulation with acetylcholine. Therefore, very low-frequency oscillations in the laser Doppler signal are NO dependent, increase as expected with cholinergic receptor-mediated stimulation, and have the potential to serve as a noninvasive marker for NO-dependent microvascular reactivity. Medium-frequency $(0.1 \mathrm{~Hz})$ spectral power has been shown to vary coherently with blood pressure and sympathetic nerve activity in diverse disease states [8-11, 23, 24].

In the present study application via microdialysis catheters of acetylcholine alone nonselectively increased spectral power across all frequency bands. If very low-frequency oscillations are specifically NO dependent, this supports the idea that cutaneous acetylcholine vasomotion as well as vasodilation are not entirely NO dependent. This is consistent with results from other tissues: thus, while acetylcholine produces significant NOdependent dilation of large systemic conduit arteries [25], NO-independent effects have also been documented [26]. The microvascular acetylcholine response may be more complex. For example, in the coronary microcirculation NO, prostaglandins, and EDHF each exert important effects [27], while in skeletal muscle NO and EDHF, but not prostaglandins, are thought to be most important [28], although results remain controversial [20]. Thus, while the direct local microvascular effects of acetylcholine are primarily endothelial, this suggests that these effects are not entirely due to nitric oxide.

While there is little doubt for a role of NO in the cutaneous response to acetylcholine [29], other vasoactive substances, such as vasodilatory or vasoconstrictive prostaglandins, may also be important to the response but remain more controversial [19, 30-32]. Our data indicate that NLA reduced acetylcholine-stimulated flow by about $50 \%$, and that NOS inhibition exerted a specific effect on very low-frequency oscillations.

Our data also demonstrate for the first time that the insertion of a microdialysis catheter does not affect cutaneous blood flow or flow spectra. This applies after a sufficient period of time is allowed for the hyperemia of catheter insertion to dissipate. Thus, future experiments using microdialysis and frequency analysis are feasible and valid.

Our work is best compared with the prior work of Kvernmo and associates, who, using wavelet analysis, first demonstrated endothelial dependence of the very low-frequency LDF oscillations using acetylcholine delivered by iontophoresis $[14,15]$. This work was further extended to study the cutaneous response to exercise [13], which suggested that oscillations near $0.1 \mathrm{~Hz}$ contribute most to the regulation of skin blood flow postexercise. Our study complements these earlier investigations by providing further evidence using NOS 
inhibition that the very low-frequency acetylcholine response does indeed represent an NOspecific response.

Also, Kvandal et al [12] previously performed studies that were similar to our present work in that the effects of the NOS inhibitor L-NMMA delivered via brachial artery had specific effects on cutaneous very low-frequency oscillations produced by acetylcholine. These were reversed by L-arginine, but were unaffected by aspirin. Differences in the route of delivery of drugs make precise comparison difficult between this and our study, which relied on intradermal drug administration through microdialysis catheters.

Clearly, the local control of cutaneous blood flow is more complex than the interplay between nitric oxide, prostaglandins (both vasodilator and vasoconstrictor), and EDHF. Also, experiments seeking to remove the effects of EDHF have yet to be performed. However, the data support a selective contribution from NO to very low-power oscillations in LDF flow-motion.

We used calves instead of forearms for purposes of microdialysis and laser Doppler flowmetry. Pilot studies show quantitative differences between measurements of LDF in arms versus legs. However, there is no qualitative difference. Thus, qualitatively similar changes occur in the forearms and calves with all drugs.

Acetylcholine may exert a neurogenic vasodilatory effect. Therefore, it might be difficult to distinguish between effects of acetylcholine on the release of $\mathrm{NO}$ and such a neurogenic effect. However, Berghoff et al., using iontophoretic administration of acetylcholine [18], showed at most a $20 \%$ reduction in the response to acetylcholine. Also, a substantial fraction of the neurogenic response can be ascribed to anodal current delivered during iontophoretic drug delivery [17]. These effects are obviated in the present study by the use of drug administration through microdialysis catheters.

Noninvasive macrovascular markers of NO such as measurements of brachial artery flowmediated dilation [33] are well known. Why add microvascular measures of NO? Arguably, endothelial dysfunction is primarily a microvascular disease and may be pre-clinically evident in the microvasculature [34]. Also, microvascular measures of endothelial function and specifically NO-dependent function may not necessarily correlate very well with macrovascular measures [35].

We used FFT rather than wavelet frequency analysis. Wavelets analysis has been used progressively more often to perform time-frequency analyses of series [13-15, 36]. While these analyses are clearly superior for nonstationary time-varying states [37], it is arguable that under steady conditions Fourier analysis and wavelet analysis may complement one another [38]. In addition, Hanning windowed FFTs have been used in the study of skin sympathetic activity [39].

Thus, using FFT, we have shown that very low-frequency oscillations in the laser Doppler signal, which increase with cholinergic receptor-mediated stimulation, may be used as a noninvasive marker of NO-dependent microvascular reactivity. 


\section{Acknowledgments}

We thank Leonard Newman and the Division of Pediatric Cardiology, especially, Michael H. Gewitz, for their unflagging support. We also thank Thomas H. Hintze, David Robertson, and Phillip Low for their constant inspiration and stimulation. This work was supported by 1RO1HL074873 from the National Heart Lung and Blood Institute and by 1R21DK071647 from the National Institute of Diabetes and Digestive and Kidney Diseases of the National Institutes of Health.

\section{REFERENCES}

1. Funk W, Endrich B, Messmer K, Intaglietta M. Spontaneous arteriolar vasomotion as a determinant of peripheral vascular resistance. Int J Microcirc Clin Exp. 1983; 2:11-25. [PubMed: 6678836]

2. Mayer S. Studien zur physiologie des herzens und der blutgefässe. V. Ueber spontane blutdruckschwankungen. Akad Wiss Wien Math Nat Kl. 1876; 74:281-307.

3. Zhang R, Iwasaki K, Zuckerman JH, Behbehani K, Crandall CG, Levine BD. Mechanism of blood pressure and R-R variability: insights from ganglion blockade in humans. J Physiol. 2002; 543:337348. [PubMed: 12181304]

4. Cohen MA, Taylor JA. Short-term cardiovascular oscillations in man: measuring and modelling the physiologies. J Physiol. 2002; 542:669-683. [PubMed: 12154170]

5. Braverman IM, Keh A, Goldminz D. Correlation of laser Doppler wave patterns with underlying microvascular anatomy. J Invest Dermatol. 1990; 95:283-286. [PubMed: 2143522]

6. Bertuglia S, Colantuoni A, Intaglietta M. Effect of leukocyte adhesion and microvascular permeability on capillary perfusion during ischemia-reperfusion injury in hamster cheek pouch. Int J Microcirc Clin Exp. 1993; 13:13-26. [PubMed: 8262718]

7. Colantuoni A, Bertuglia S, Intaglietta M. Microvascular vasomotion: origin of laser Doppler flux motion. Int J Microcirc Clin Exp. 1994; 14:151-158. [PubMed: 8082994]

8. Bernardi L, Hayoz D, Wenzel R, Passino C, Calciati A, Weber R, Noll G. Synchronous and baroceptor-sensitive oscillations in skin microcirculation: evidence for central autonomic control. Am J Physiol. 1997; 273:H1867-H1878. [PubMed: 9362255]

9. Soderstrom T, Stefanovska A, Veber M, Svensson H. Involvement of sympathetic nerve activity in skin blood flow oscillations in humans. Am J Physiol Heart Circ Physiol. 2003; 284:H1638-H1646. [PubMed: 12679328]

10. Furlan R, Porta A, Costa F, Tank J, Baker L, Schiavi R, Robertson D, Malliani A, MosquedaGarcia R. Oscillatory patterns in sympathetic neural discharge and cardiovascular variables during orthostatic stimulus. Circulation. 2000; 101:886-892. [PubMed: 10694528]

11. Cogliati C, Magatelli R, Montano N, Narkiewicz K, Somers VK. Detection of low- and highfrequency rhythms in the variability of skin sympathetic nerve activity. Am J Physiol Heart Circ Physiol. 2000; 278:H1256-H1260. [PubMed: 10749722]

12. Kvandal P, Stefanovska A, Veber M, Kvernmo HD, Kirkeboen KA. Regulation of human cutaneous circulation evaluated by laser Doppler flowmetry, iontophoresis, and spectral analysis: importance of nitric oxide and prostaglandines. Microvasc Res. 2003; 65:160-171. [PubMed: 12711257]

13. Kvernmo HD, Stefanovska A, Bracic M, Kirkeboen KA, Kvernebo K. Spectral analysis of the laser Doppler perfusion signal in human skin before and after exercise. Microvasc Res. 1998; 56:173-182. [PubMed: 9828155]

14. Kvernmo HD, Stefanovska A, Kirkeboen KA, Kvernebo K. Oscillations in the human cutaneous blood perfusion signal modified by endothelium-dependent and endothelium-independent vasodilators. Microvasc Res. 1999; 57:298-309. [PubMed: 10329256]

15. Kvernmo HD, Stefanovska A, Kirkeboen KA. Enhanced endothelial activity reflected in cutaneous blood flow oscillations of athletes. Eur J Appl Physiol. 2003; 90:16-22. [PubMed: 12783233]

16. Durand S, Fromy B, Bouye P, Saumet JL, Abraham P. Vasodilatation in response to repeated anodal current application in the human skin relies on aspirin-sensitive mechanisms. J Physiol. 2002; 540:261-269. [PubMed: 11927685] 
17. Tartas M, Durand S, Koitka A, Bouye P, Saumet JL, Abraham P. Anodal current intensities above 40 microA interfere with current-induced axon-reflex vasodilatation in human skin. J Vasc Res. 2004; 41:261-267. [PubMed: 15153776]

18. Berghoff M, Kathpal M, Kilo S, Hilz MJ, Freeman R. Vascular and neural mechanisms of AChmediated vasodilation in the forearm cutaneous microcirculation. J Appl Physiol. 2002; 92:780 788. [PubMed: 11796692]

19. Kellogg DL Jr, Zhao JL, Coey U, Green JV. Acetylcholine-induced vasodilation is mediated by nitric oxide and prostaglandins in human skin. J Appl Physiol. 2005; 98:629-632. [PubMed: 15649880]

20. de Wit C, Esser N, Lehr HA, Bolz SS, Pohl U. Pentobarbital-sensitive EDHF comediates AChinduced arteriolar dilation in the hamster microcirculation. Am J Physiol. 1999; 276:H1527H1534. [PubMed: 10330235]

21. Anderson C, Andersson T, Wardell K. Changes in skin circulation after insertion of a microdialysis probe visualized by laser Doppler perfusion imaging. J Invest Dermatol. 1994; 102:807-811. [PubMed: 8176267]

22. Stewart JM, Medow MS, Cherniack NS, Natelson BH. Postural hypocapnic hyperventilation is associated with enhanced peripheral vasoconstriction in postural tachycardia syndrome with normal supine blood flow. Am J Physiol Heart Circ Physiol. 2006; 291:H904-H913. [PubMed: 16565300]

23. Lefrandt JD, Bosma E, Oomen PH, Hoeven JH, Roon AM, Smit AJ, Hoogenberg K. Sympathetic mediated vasomotion and skin capillary permeability in diabetic patients with peripheral neuropathy. Diabetologia. 2003; 46:40-47. [PubMed: 12637981]

24. Wilson TE, Zhang R, Levine BD, Crandall CG. Dynamic autoregulation of cutaneous circulation: differential control in glabrous versus nonglabrous skin. Am J Physiol Heart Circ Physiol. 2005; 289:H385-H391. [PubMed: 15749747]

25. Stoen R, Lossius K, Karlsson JO. Acetylcholine-induced vasodilation may depend entirely upon NO in the femoral artery of young piglets. Br J Pharmacol. 2003; 138:39-46. [PubMed: 12522071]

26. Ming Z, Parent R, Lavallee M. Nitric oxide-independent dilation of conductance coronary arteries to acetylcholine in conscious dogs. Circ Res. 1997; 81:977-987. [PubMed: 9400378]

27. Nishikawa Y, Stepp DW, Chilian WM. Nitric oxide exerts feedback inhibition on EDHF-induced coronary arteriolar dilation in vivo. Am J Physiol Heart Circ Physiol. 2000; 279:H459-H465. [PubMed: 10924042]

28. Huang A, Sun D, Smith CJ, Connetta JA, Shesely EG, Koller A, Kaley G. In eNOS knockout mice skeletal muscle arteriolar dilation to acetylcholine is mediated by EDHF. Am J Physiol Heart Circ Physiol. 2000; 278:H762-H768. [PubMed: 10710344]

29. Boutsiouki P, Georgiou S, Clough GF. Recovery of nitric oxide from acetylcholine-mediated vasodilatation in human skin in vivo. Microcirculation. 2004; 11:249-259. [PubMed: 15280079]

30. Holowatz LA, Thompson CS, Minson CT, Kenney WL. Mechanisms of acetylcholine-mediated vasodilatation in young and aged human skin. J Physiol. 2005; 563:965-973. [PubMed: 15661816]

31. Durand S, Tartas M, Bouye P, Koitka A, Saumet JL, Abraham P. Prostaglandins participate in the late phase of the vascular response to acetylcholine iontophoresis in humans. J Physiol. 2004; 561:811-819. [PubMed: 15498811]

32. Dalle-Ave A, Kubli S, Golay S, Delachaux A, Liaudet L, Waeber B, Feihl F. Acetylcholineinduced vasodilation and reactive hyperemia are not affected by acute cyclo-oxygenase inhibition in human skin. Microcirculation. 2004; 11:327-336. [PubMed: 15280072]

33. Celermajer DS, Sorensen KE, Gooch VM, Miller OI, Spiegelhalter DJ, Sullivan ID, Lloyd JK, Deanfield JE. Non-invasive detection of endothelial dysfunction in children and adults at risk of atherosclerosis. Lancet. 1992; 340:1111-1115. [PubMed: 1359209]

34. Creager MA, Cooke JP, Mendelsohn ME, Gallagher SJ, Coleman SM, Loscalzo J, Dzau VJ. Impaired vasodilation of forearm resistance vessels in hypercholesterolemic humans. J Clin Invest. 1990; 86:228-234. [PubMed: 2195060]

35. Agewall S, Henareh L, Kublickiene K. Endothelial function in conduit and resistance arteries in men with coronary disease. Atherosclerosis. 2006; 184:130-136. [PubMed: 15979080] 
36. Jan YK, Brienza DM, Geyer MJ. Analysis of week-to-week variability in skin blood flow measurements using wavelet transforms. Clin Physiol Funct Imaging. 2005; 25:253-262. [PubMed: 16117727]

37. Belova NY, Mihaylov SV, Piryova BG. Wavelet transform: a better approach for the evaluation of instantaneous changes in heart rate variability. Auton. Neurosci. 2007; 131:107-122. [PubMed: 16942920]

38. Jimenez RF, Torres P, Gunther B, Morgado E, Jimenez CA. Wavelet and Fourier analysis of ventricular and main arteries pulsations in anesthetized dogs. Biol Res. 2004; 37:431-447. [PubMed: 15515968]

39. Cui J, Sathishkumar M, Wilson TE, Shibasaki M, Davis SL, Crandall CG. Spectral characteristics of skin sympathetic nerve activity in heat-stressed humans. Am J Physiol Heart Circ Physiol. 2006; 290 

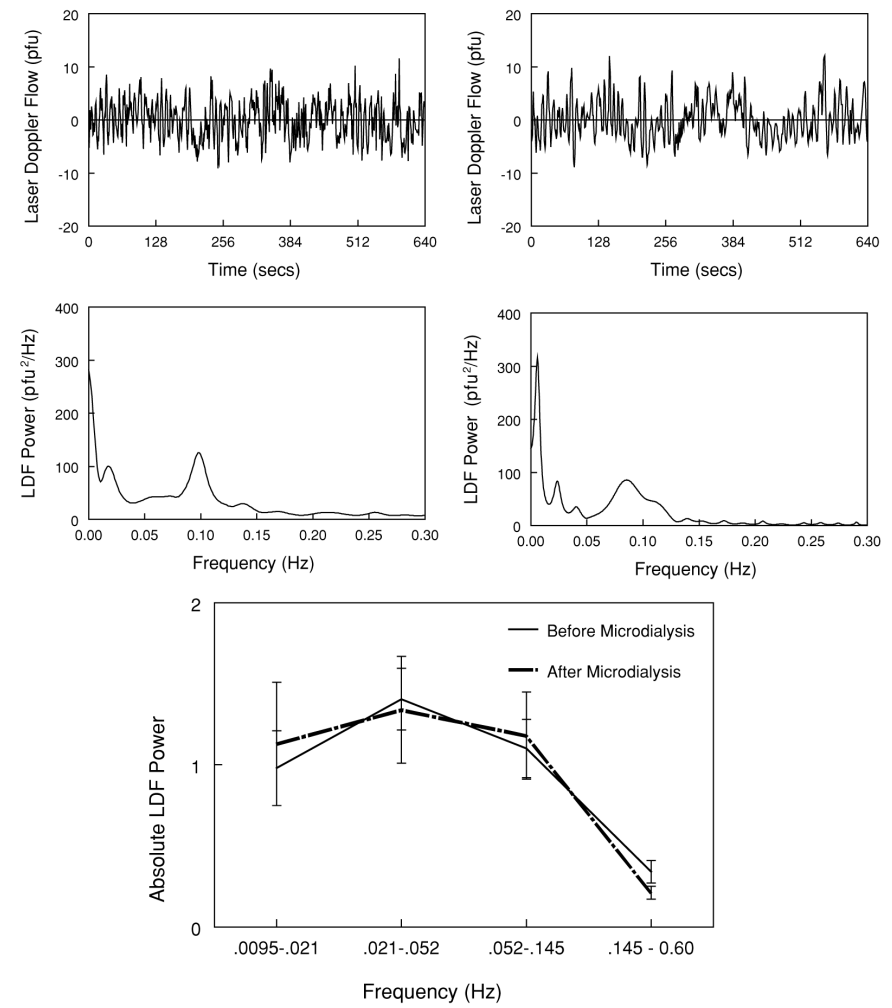

Figure 1.

(Top) Laser Doppler flow (LDF) and corresponding power spectrum before (left) and after (right) insertion of the microdialysis catheters. Patients recovered from the insertion procedure for at least $1 \mathrm{~h}$. (Bottom) The absolute summed laser Doppler power in each frequency band. There is no significant difference before or after recovery from microdialysis probe insertion. VLF $=0.0095-0.021 \mathrm{~Hz}, \mathrm{LF}=0.021-0.052 \mathrm{~Hz}, \mathrm{MF}=0.052-$ $0.145 \mathrm{~Hz}, \mathrm{HF}=0.145-0.6 \mathrm{~Hz}$. 

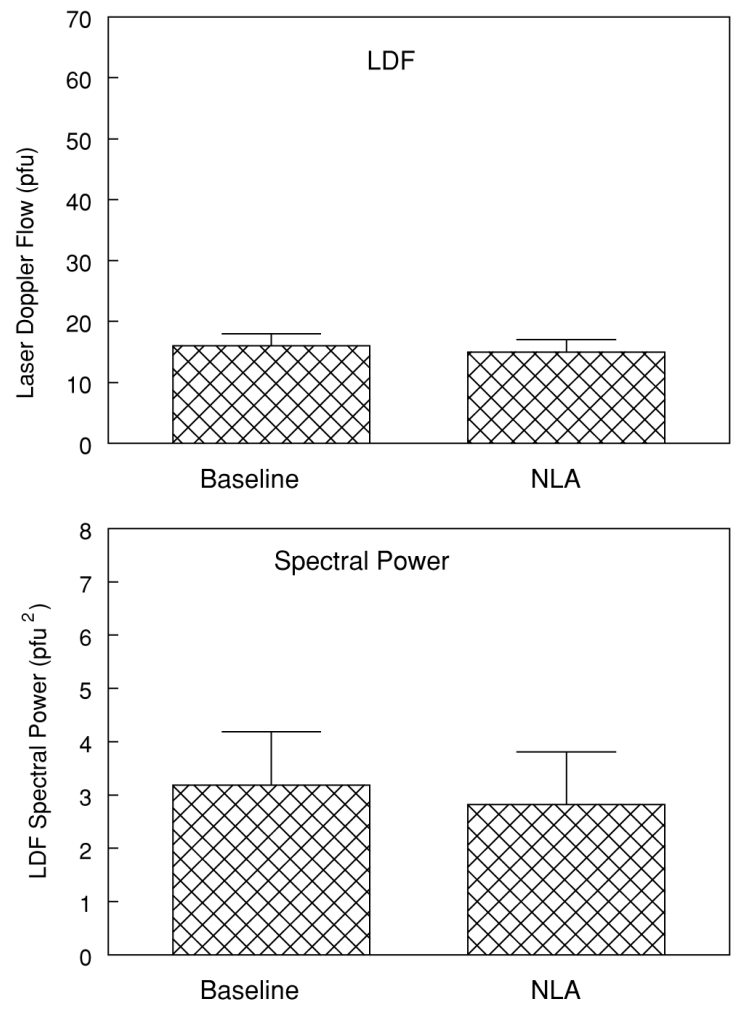

Figure 2.

The effects of NLA on absolute laser Doppler blood flows (LDF), expressed in perfusion units (pfu in the upper panels), and total spectral power summed over all frequency bands, (pfu2 in the lower panels). Comparison is between the baseline determined after the microdialysis probe has been inserted and after NLA is given. No differences in laser Doppler flow or total power are evident. 

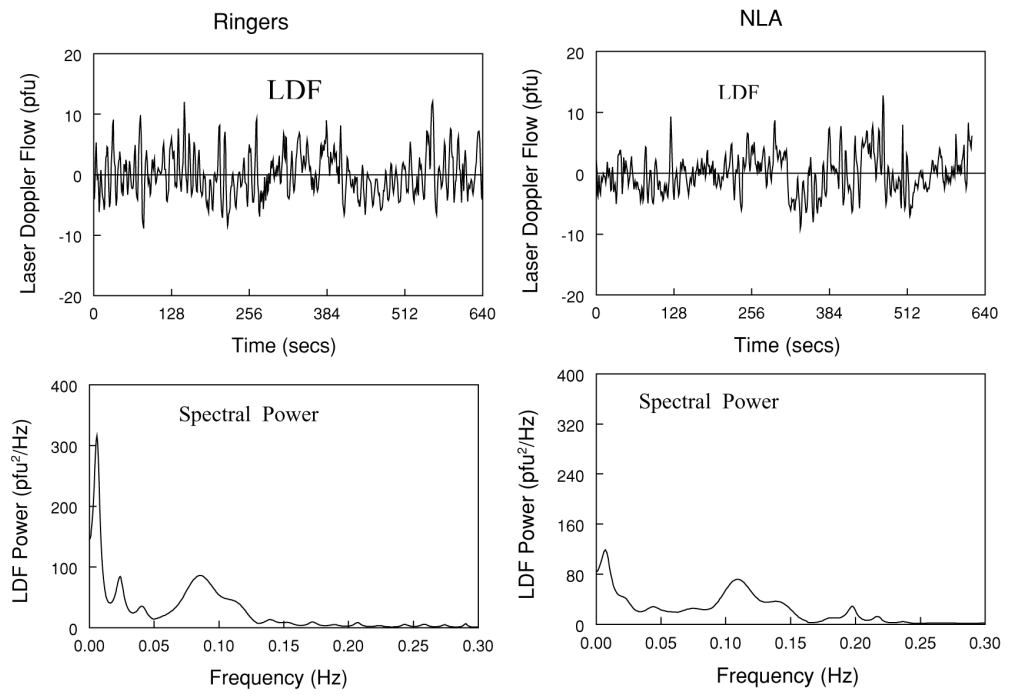

Figure 3.

Representative LDF and corresponding power spectra: (Left) Results during perfusion with Ringer's solution; (right) results during perfusion with 10 mM NLA dissolved in Ringer's solution. There is marked decrease in very low-frequency power. VLF $=0.0095-0.021 \mathrm{~Hz}$, $\mathrm{LF}=0.021-0.052 \mathrm{~Hz}, \mathrm{MF}=0.052-0.145 \mathrm{~Hz}, \mathrm{HF}=0.145-0.6 \mathrm{~Hz}$. 

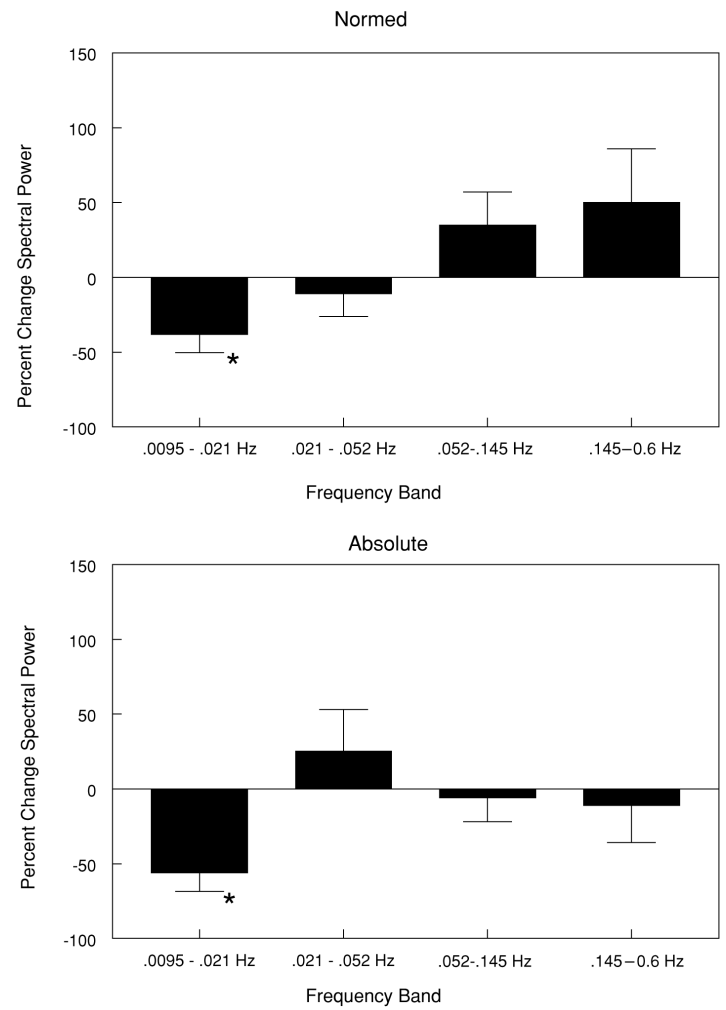

Figure 4.

The effects of NLA on spectral power at each frequency band. Percent changes in spectral power from baseline are shown. (Top) Changes in data normalized to total power. (Bottom) Changes in absolute spectral power. Very low-frequency power is only and exclusively reduced following the addition of NLA. $* p<.05$ compared to baseline. VLF $=0.0095-$ $0.021 \mathrm{~Hz}, \mathrm{LF}=0.021-0.052 \mathrm{~Hz}, \mathrm{MF}=0.052-0.145 \mathrm{~Hz}, \mathrm{HF}=0.145-0.6 \mathrm{~Hz}$. 

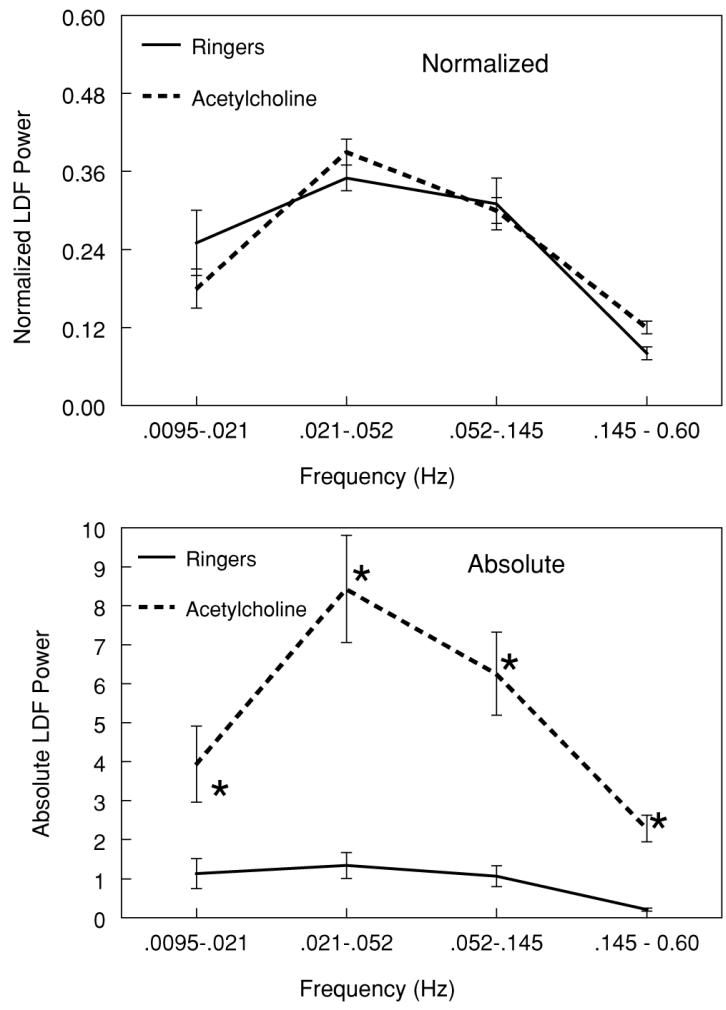

Figure 5.

The effects of acetylcholine $(100 \mathrm{mM})$ on laser Doppler flow in all subjects $(N=22)$. (Top) Data normalized to total power (the sum of the power in all frequency bands). (Bottom) The absolute spectral power in units of pfu2. Acetylcholine markedly increases absolute power compared to baseline (Ringer solution) but normalized power is unaffected. This implies that power in each frequency band is increased by the same fraction. $* p<.05$ compared to Ringer's solution. VLF $=0.0095-0.021 \mathrm{~Hz}, \mathrm{LF}=0.021-0.052 \mathrm{~Hz}, \mathrm{MF}=0.052-0.145 \mathrm{~Hz}$, $\mathrm{HF}=0.145-0.6 \mathrm{~Hz}$. 
LDF U/A Spectral Power

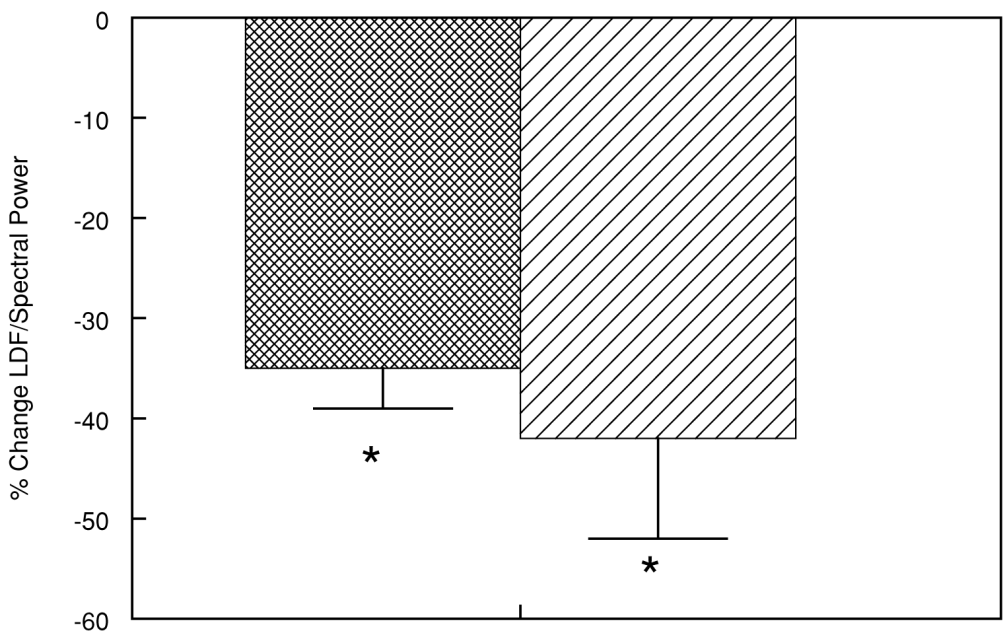

Figure 6.

The effects of NLA during concurrent acetylcholine ( $\mathrm{AcCh}$ ) infusion on percent change in laser Doppler flow (LDF) and total spectral power compared to acetylcholine alone summed over all frequency bands. Addition of NLA to acetylcholine marked reduces flow and spectral power. $* p<.05$ compared to acetylcholine alone. 

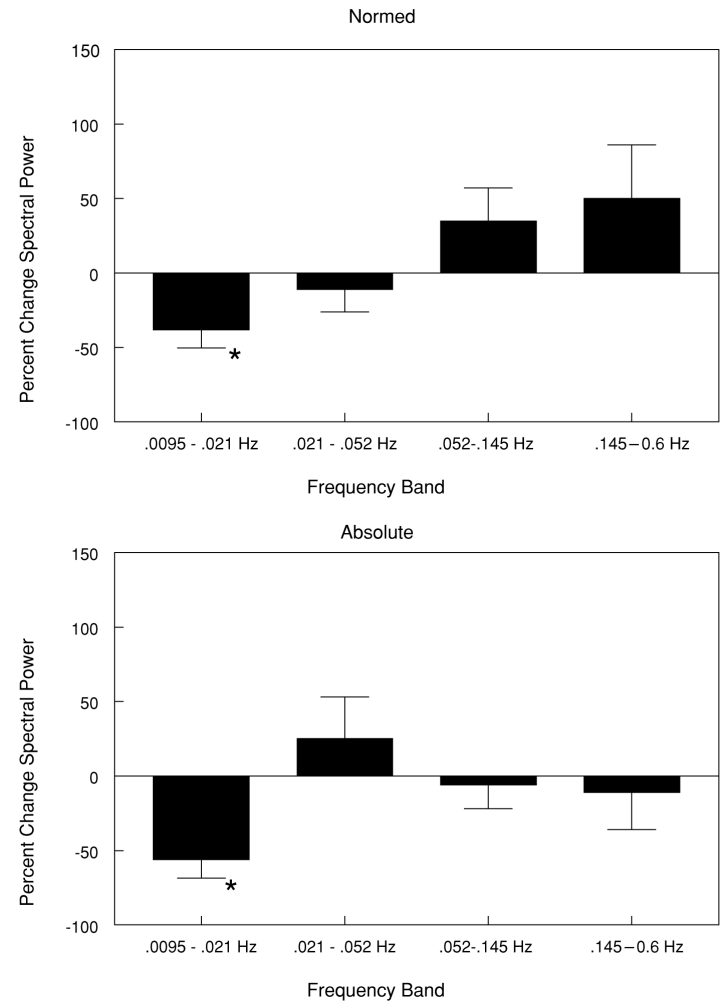

Figure 7.

The effects of NLA on spectral power at each frequency band during concurrent acetylcholine infusion. Percent changes from acetylcholine alone are shown. (Top) Changes in data normalized to total power. (Bottom) Changes in absolute spectral power. Very lowfrequency power is reduced following the addition of NLA. $* p<.05$ compared to acetylcholine alone. VLF $=0.0095-0.021 \mathrm{~Hz}, \mathrm{LF}=0.021-0.052 \mathrm{~Hz}, \mathrm{MF}=0.052-0.145 \mathrm{~Hz}$, $\mathrm{HF}=0.145-0.6 \mathrm{~Hz}$. 